\title{
EL IMPACTO DEL DERECHO Y EL PENSAMIENTO JURÍDICO DE LOS ESTADOS UNIDOS: ¿AMENAZA O INCENTIVO?*
}

\author{
Ewoud Hondius**
}

RESUMEN

El presente trabajo analiza la influencia que el derecho y la doctrina jurídica de los Estados Unidos ejerce actualmente sobre la academia y legislación de otros países o sistemas jurídicos, exponiendo las razones de tal predominio y proponiendo como principales medidas para contrarrestar dicha tendencia, la acción coordinada a través de organizaciones regionales como la Unión Europea y la neutralización del idioma inglés como lengua franca universal.

DERECHO DE LOS ESTADOS UNIDOS - DOCTRINA JURÍDICA DE LOS ESTADOS UNIDOS - INFLUENCIA DEL DERECHO NORTEAMERICANO

\section{The impact of American law and American legal theory: threat or incentive?}

\begin{abstract}
The article analyzes the impact of A merican law and legal theory on the law and legal doctrine of other jurisdictions, examining the reasons for such influence and stating that the way to oppose that tendency is through the coordinated action of regional organizations such as the European Union and neutralizing the English language as the world's lingua franca.
\end{abstract}

\section{AMERICAN LAW - AMERICAN LEGAL THINKING - IMPACT OF AMERICAN LAW}

* El presente trabajo es una versión ampliada y actualizada del artículo publicado originalmente bajo el título de "The Impact of American Law and American Legal Theory: Threat or Incentive?", en B. FauvarqueCosson (Ed.), De tous horizons/Mélanges Xavier Blanc-Jouvan, Societé de législation comparée, Paris, 2005, pp. 271-280. La traducción al español ha sido realizada por Rodrigo Momberg Uribe, Profesor de Derecho Civil de la Facultad de Ciencias Jurídicas y Sociales de la Universidad Austral de Chile.

** Abogado, Doctor en Derecho, Profesor de Derecho en el Molengraaff Institute of Private Law, Universidad de Utrecht, Nobelstraat 2a, 3512 EN, Utrecht, Holanda. e.h.hondius@uu.nl

Artículo recibido el 28 de agosto de 2009 y aceptado para su publicación por el Comité Editorial el 26 de octubre de 2009. 


\section{INTRODUCCIÓN}

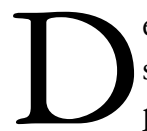
esde mediados del siglo pasado, han sido muchos quienes han optado por cursar sus estudios de postgrado en Derecho en los Estados Unidos de América. ${ }^{1}$ Gran parte de estos estudiantes son atraídos por la calidad académica de las universidades del referido país, y quizás, en mayor medida, por el impacto y la influencia que el derecho y el pensamiento jurídico norteamericano tienen en el mundo contemporáneo. Mi objetivo en el presente trabajo no es examinar las consecuencias que tal impacto tiene en la práctica jurídica diaria, como por ejemplo si es más conveniente que ciertos contratos se sujeten al derecho inglés o al del estado de Nueva York, o si el Presidente de los Estados Unidos tiene el poder para forzar un procedimiento criminal en contra de algún líder de una potencia antagonista a los intereses norteamericanos (como ocurrió en el caso de Saddam Hussein). Al contrario, lo que pretendo analizar a continuación es la influencia que el derecho y la doctrina jurídica norteamericana ejercen actualmente sobre la academia y legislación de otros países o sistemas jurídicos.

Los argumentos que desarrollo en este trabajo tienen como antecedente un artículo anterior, ${ }^{2}$ en que cuestionaba la idea de la supremacía del derecho occidental (expresado a través de los diversos sistemas jurídicos que lo componen), creencia predominante hoy en día y que parece ser la razón para que la gran mayoría de los estudiantes graduados en Derecho prosigan sus estudios de Magíster en Europa, Norteamérica o Australia. Inspirado en la obra de Patrick Glenn, ${ }^{3}$ refutaba esa idea y en cambio planteaba que es mucho lo que se puede aprender del derecho de los países del tercer mundo.

Sin embargo, aunque relacionado con el anterior, mi propósito ahora es analizar específicamente el impacto del derecho y el pensamiento jurídico norteamericano en el mundo occidental. En un artículo publicado el año 2003, Ugo Mattei desarrolló un tema similar, pero con objetivos distintos. ${ }^{4}$ En dicho trabajo, Mattei plantea que "el derecho imperialista es hoy en día el componente dominante en los sistemas jurídicos a nivel mundial. El derecho imperialista es generado, en torno al interés de capitales internacionales, por una serie de entidades públicas y privadas, todas compartiendo un vacío de legitimidad, denominado a veces 'déficit democrático'. Este derecho imperialista ha sido configurado por un espectacular proceso de exageración, destinado a construir consenso con el propósito de conseguir una posición hegemónica. Con este fin, el derecho imperialista concluye acuerdos con los derechos locales, reproduciendo a nivel global el fenómeno de dualismo jurídico que hasta ahora ha caracterizado el derecho de los países en desarrollo. Una predatoria globalización económica es el vehículo, la aliada

${ }^{1}$ En este trabajo, por razones de estilo, se utilizarán las expresiones Estados Unidos y Norteamérica, y consecuentemente estadounidense y norteamericano, como sinónimos (nota del traductor).

2 "The Supremacy of Western Law", en C. de Ligt et al. (Ed.), Viva vox iuris Romani/Essays in honour of Jobannes Emil Spruit, Gieben, Amsterdam 2002 pp. 337-342.

${ }^{3}$ Glenn, H. P., Legal Traditions of the World, Oxford U.P., Oxford, 2004.

${ }^{4}$ Mattei, U., "A Theory of Imperial Law: A Study on U.S. Hegemony and the Latin Resistance”, Global Jurist Frontiers, vol. 3, issue 2, (2003), disponible en http://www.bepress.com/gj/frontiers/vol3/iss2/art1/\# 
todopoderosa y beneficiaria del derecho imperialista. Irónicamente, a pesar de su absoluta falta de legitimidad democrática, este derecho imperialista impone, como una necesidad natural y por medio de prácticas retóricas, aquello que se ha denominado 'democracia y estado de derecho', esto es, una filosofía reaccionaria, contraria a la redistribución de la riqueza basada en ideas de solidaridad social". 5

¿En qué se relacionan estos planteamientos con el derecho norteamericano? Mattei aclara que "en la esencia del derecho imperialista está el derecho de los Estados Unidos, tal como ha sido transformado y adaptado después de la revolución Reagan-Thatcher, en el proceso de infiltrar la vasta periferia abierta tras el término de la guerra fría". 6 Aun cuando el trabajo de Mattei tiene propósitos muy distintos a los que pretendo desarrollar, algunos de sus planteamientos sirven de base para el planteamiento de mi hipótesis.

Tal hipótesis es, en términos breves, que Norteamérica, y en particular los Estados Unidos, ha sido extremadamente exitoso en la difusión de su derecho y su doctrina jurídica. ${ }^{7}$ Sin embargo, mi planteamiento es que organizaciones regionales (y no países o jurisdicciones de manera aislada) pueden servir como contrapeso a esta tendencia. Una de las más exitosas organizaciones regionales a este respecto es la Unión Europea, pero otras organizaciones como el Pacto Andino, ASEAN (Association of Southeast Asia Nations), MERCOSUR y OHADA (Organisation pour l'Harmonisation en Afrique du Droit des Affaires), por mencionar algunas, pueden también ser actores relevantes en el futuro.

Hasta este punto podría pensarse que los Estados Unidos son el gran enemigo y que todo abogado o jurista no estadounidense debería movilizarse para combatir este enemigo común. Sin embargo, mi planteamiento es muy distinto. Al contrario, los Estados Unidos deberían, en primer lugar, ser reconocidos por la preeminencia que le han otorgado al derecho y a la teoría jurídica. Sus escuelas de derecho, como Berkeley, Columbia, Harvard, NYU, Yale, entre otras, están insertas en instituciones de enseñanza de nivel mundial. A su vez, la doctrina norteamericana ha sido exitosa en generar y proveer al mundo de importantes e innovadoras teorías jurídicas. El realismo jurídico, las doctrinas jurídicas sobre discriminación y derecho, sobre género y derecho, la escuela del estudio crítico del derecho y el análisis económico del derecho son algunos ejemplos obvios. Tales teorías son producto de diseños curriculares extremadamente motivantes que incitan a los académicos a desarrollar nuevas ideas en lugar de sumergir a sus audiencias en el mero análisis de los casos que los mismos académicos en su calidad de abogados conocen en su vida profesional.

Justo también es reconocer las influencias europeas que han ayudado al derecho norteamericano a ocupar su destacado rol en el mundo contemporáneo. Al respecto,

${ }^{5}$ Mattei, op. cit., p. 1.

${ }^{6}$ Ibid., p. 2.

7 "Las teorías jurídicas predominantes, generadas hasta hace un tiempo en el derecho continental europeo y exportadas hacia la periferia del mundo, son ahora, por primera vez, generadas en una jurisdicción perteneciente al common law: los Estados Unidos”, Mattei, op. cit., p. 2. 
Mattei menciona la influencia de tres grandes tradiciones jurídicas en el desarrollo del derecho estadounidense: 8

En primer lugar, lógicamente, el common law inglés, que constituye la base del sistema jurídico norteamericano y de su poder judicial fuerte e independiente.

Segundo, la concepción francesa de derechos individuales de carácter universal, consagrada en la Constitución de los Estados Unidos, y

Tercero, la tradición alemana de enseñar el derecho en instituciones académicas prestigiosas e independientes. A este respecto, hay que destacar que desde temprano los Estados Unidos se alejaron de la tradición del common law inglés, según la cual (hasta avanzado el siglo XIX) el derecho no era considerado como objeto merecedor de análisis académico, sino tan sólo una profesión que se adquiría a través de la práctica.

\section{TEORÍA JURÍdiCAS NORTEAMERICANAS QUE HAN CONQUISTADO EL MUNDO}

Antes de analizar algunas de las razones por las que el derecho norteamericano es hoy en día tan exitoso, creo conveniente desarrollar con mayor detalle algunos de los ejemplos mencionados en el apartado anterior. Los casos de las teorías o doctrinas sobre discriminación y derecho (a), de la responsabilidad por productos defectuosos (b), del análisis económico del derecho (c), del estudio crítico del derecho (d) y de la mediación (e) serán analizadas a continuación. Hay por cierto, numerosos otros ejemplos, así como algunos fracasos, uno de los cuales es el caso del realismo jurídico (f).

a) Inspirados por la doctrina de los derechos humanos, los norteamericanos han combatido la discriminación en variadas formas. Brown v. Board of Education ${ }^{9}$ es todavía un caso esencial para el desarrollo del derecho contra la discriminación racial. Lo mismo sucede con Roev. Wade ${ }^{10}$ en relación con la discriminación por género. En estas materias, las publicaciones norteamericanas han tenido (y tienen) gran relevancia en la formación y desarrollo de la doctrina de los derechos humanos. Por otra parte, estas doctrinas no sólo han tenido impactos sociales, sino también ha modificado la estructura del derecho. El derecho se ha constitucionalizado. Así, actualmente sólo en un número menor de jurisdicciones no se admite aún el control o la revisión constitucional por parte de tribunales superiores de justicia o cortes especializadas.

b) En derecho privado, el desarrollo de la responsabilidad por productos defectuosos es una creación norteamericana. Esta doctrina ha sido acogida favorablemente por la mayoría de la comunidad jurídica mundial. Incluso, de alguna manera, puede decirse que los Estados Unidos han sido derrotados por su propio éxito. Así, en Europa,

\footnotetext{
${ }^{8}$ Ibid., p. 7.

9347 U.S. 483 (1954).

10410 U.S. 113 (1973).
} 
la Directiva sobre Productos Defectuosos ${ }^{11}$ ha sido implementada en todos los estados miembros, y ha servido de modelo para la legislación de muchos otros países, incluyendo Australia y Japón. ${ }^{12}$ Sin perjuicio de lo anterior, es evidente que la idea de promover la responsabilidad por productos defectuosos como una institución jurídica autónoma es de origen estadounidense.

c) Richard Posner es el padre fundador de la escuela del análisis económico del derecho, la cual ha tenido un éxito universal, al punto que hoy en día muchas discusiones jurídicas y políticas se plantean principalmente sobre la base de argumentos económicos y no éticos. ${ }^{13}$ La amplia recepción que esta teoría ha tenido fuera de las fronteras de los Estados Unidos puede ser ejemplificada por el hecho que en una Facultad de Derecho como la de la Universidad de Utrecht, uno de los programas de Magíster más exitosos entre los estudiantes extranjeros es justamente aquel referido al análisis económico del derecho.

d) El estudio crítico del derecho es también una doctrina que tiene su origen en los Estados Unidos. ${ }^{14}$ El impacto que ha tenido en otras partes del mundo puede ser ilustrado por la organización de un seminario llevado a cabo en Amsterdam el año 2000, dedicado a 'El estudio crítico del derecho y el derecho privado europeo'. ${ }^{15}$ Tal como en el caso del análisis económico del derecho, el estudio crítico del derecho es obra principalmente de una persona, Duncan Kennedy. ${ }^{16}$ Sin embargo, esta teoría ha sido menos exitosa en cuanto a crear una infraestructura que la sustente, consistente en estos casos en cátedras universitarias, revistas especializadas y asociaciones nacionales e internacionales dedicadas al tema.

e) Los métodos de resolución alternativa de conflictos, y en especial la mediación, también han sido generados desde los Estados Unidos, como herramientas efectivas para prevenir y resolver controversias. En todo caso, debe aclararse que la mediación no es estrictamente de origen norteamericano. Así, durante la Revolución Francesa, la mediación adquirió gran relevancia, y en materias de familia era la regla y no la excepción a principios del siglo XIX en Francia. Sin embargo, fue en Estados Unidos donde la mediación

${ }^{11}$ Directiva 85/374/CEE del Consejo, de 25 de julio de 1985, relativa a la aproximación de las disposiciones legales, reglamentarias y administrativas de los Estados miembros en materia de responsabilidad por los daños causados por productos defectuosos.

${ }^{12}$ Véase, sin embargo, Reimann M., "Product Liability in a Global Context: The Hollow Victory of the European Model", en European Review of Private Law, 2003, p. 128-154, quien señala que "debería reconocerse no sólo lo exitoso que ha sido el modelo europeo en cuanto a la adopción de legislación en el resto del mundo, sino también cuán poco eso ha significado en la práctica” (p. 153).

${ }^{13}$ Mattei agrega que el análisis económico del derecho es actualmente una de las doctrinas académicas más influyentes dentro del Banco Mundial y del Fondo Monetario Internacional. Mattei, op. cit., p. 25.

${ }^{14}$ La idea básica del estudio crítico del derecho es que el derecho no es un sistema neutral ni objetivo cuyo fin último es la justicia, sino la manifestación política de las diversas relaciones de poder que existen en la sociedad y, en definitiva, un instrumento para el mantenimiento de tales jerarquías.

${ }^{15} \mathrm{Al}$ efecto, véase el número especial de la European Review of Private Law 2002, sobre "Estudio Crítico del Derecho".

${ }^{16}$ A Critique of Adjudication (fin de siècle), Cambridge, Mass., 1997, de Dunkan Kennedy, es considerada como la obra fundacional de la escuela del estudio crítico del derecho. 
adquirió popularidad como mecanismo de resolución de conflictos, debido principalmente a la necesidad de economizar costos en materia de litigación. Un caso, tomado de mi experiencia como juez, puede servir de ejemplo para demostrar lo anterior. En éste, uno de los contratantes había demandado paralelamente a su contraparte y al asegurador de dicha contraparte, desarrollándose uno de los juicios en los Estados Unidos y el otro en Holanda. El procedimiento seguido ante el tribunal norteamericano destacaba por su alta calidad técnica. Por ejemplo, los informes relativos a las declaraciones de testigos eran excelentes. En cambio, el procedimiento seguido ante los tribunales holandeses era de una calidad inferior. Sin embargo, el costo de este último era aproximadamente 30.000 florines (unos 14.000 euros), mientras que los costos del procedimiento en los Estados Unidos alcanzaban el millón de dólares. Es evidente que ante tales costos, la necesidad de medios alternativos a la litigación ante tribunales ordinarios es mucho mayor que en el caso de países del sistema de derecho civil. Actualmente, la importancia de la mediación como medio de resolución de conflictos en materias civiles y comerciales ha sido reconocida y regulada en la Unión Europea a través de una Directiva. ${ }^{17}$

f) Sin embargo, no todas las teorías jurídicas provenientes de los Estados Unidos han sido exitosas a nivel internacional. Un ejemplo de ello es la del realismo norteamericano, el cual sin embargo su éxito en los Estados Unidos no ha logrado la misma trascendencia en otras jurisdicciones. ${ }^{18}$ Así, los juristas extranjeros frecuentemente se sorprenden del interés que los estudios empíricos y sociológicos tienen para los académicos norteamericanos. Por tal motivo, 'la primera reacción de un Europeo al hojear una revista jurídica norteamericana, es decir que ella casi no contiene artículos de derecho'. ${ }^{19}$

\section{Cómo los Estados Unidos han CONQuistado El MUNdo JURídico}

¿De qué manera los Estados Unidos han logrado todo esto? ¿Es porque dicho país es la mayor potencia económica mundial? ¿Es debido a su preponderancia política y militar? Es cierto que los Estados Unidos, a través de organismos en los que tiene participación importante, como el Banco Mundial, el Fondo Monetario Internacional y la Organización Mundial de Comercio ha presionado en determinadas ocasiones a países del Tercer Mundo a aceptar sus modelos jurídicos 'como parte de sutiles chantajes' ${ }^{20}$ Sin embargo, ninguno de tales argumentos es enteramente convincente. Japón, por ejemplo, es sin duda uno de los países más importantes en la economía mundial. A pesar de ello, el derecho japonés y, por tanto, su doctrina jurídica, no han alcanzado ni remotamente la relevancia del derecho norteamericano.

${ }^{17}$ Directiva 2008/52/CE del Parlamento Europeo y del Consejo, de 21 de mayo de 2008, sobre ciertos aspectos de la mediación en asuntos civiles y mercantiles.

${ }^{18}$ Hesselink, M. W., The New European Private Law/Essays on the Future of Private Law, The Hague, Kluwer, 2002, p. 22 y sgtes.

${ }^{19}$ Hesselink, op. cit., p. 26

${ }^{20}$ Mattei, op. cit., p. 6. 
Por otra parte, el argumento que funda la supremacía jurídica norteamericana en su poderío militar y político es más difícil de rebatir. En la época de la Guerra Fría, si bien Estados Unidos era una de las superpotencias, su contraparte, la Unión Soviética, ejerció también una vasta e importante influencia. Marxismo, estalinismo y comunismo, como ideologías políticas y jurídicas, fueron implantados en muchos países del ámbito socialista. Dicho impacto, sin embargo, fue limitado temporal e ideológicamente, en el sentido que después de la desaparición de los sistemas socialistas tales doctrinas fueron rápidamente descartadas, siendo importadas para su implementación nuevas leyes y teorías jurídicas provenientes de Occidente.

Lo que sin embargo debe tenerse en cuenta es que aún sin considerar su poder económico, político y militar, el derecho y el pensamiento jurídico de los Estados Unidos ha transcendido a nivel mundial debido a su intrínseca fortaleza. ${ }^{21}$ Sin pretender un análisis acabado del tema, al menos cinco elementos pueden distinguirse a este efecto: a) la calidad de la educación jurídica norteamericana; b) el hecho de que los estadounidenses hablan inglés; c) la forma de presentación de sus ideas; d) el número de abogados norteamericanos, y e) el hecho que los Estados Unidos son un estado federal y, por tanto, descentralizado.

a) Los Estados Unidos han sido capaces de crear y sustentar en el tiempo grandes instituciones de enseñanza. El vínculo con los estudiantes se mantiene mucho después que éstos han abandonado las aulas. Cualquiera que haya estudiado derecho en una Universidad norteamericana se habrá dado cuenta hasta qué grado tales universidades buscan mantener las relaciones con sus ex alumnos. Lo anterior es no sólo un asunto de recolección de fondos (aun cuando ello es uno de sus principales propósitos) sino también de mantener a sus antiguos alumnos comprometidos con la institución. Por otra parte, la afluencia de estudiantes extranjeros a sus programas de pre y postgrado ha colaborado a elevar la calidad global del currículum en las escuelas de derecho norteamericanas.

b) Los académicos norteamericanos poseen la ventaja obvia de tener como lengua materna al idioma inglés, actualmente la lingua franca universal. Ello les permite intervenir en el discours juridique sin ningún tipo de restricción lingüística y, aún más, les otorga una ventaja en el debate intelectual frente a académicos de origen distinto al anglosajón.

c) Los académicos norteamericanos, más que sus pares ingleses, ${ }^{22}$ se han convertido en maestros del estilo. Pueden darse dos ejemplos de lo anterior. Primero, las revistas de derecho de origen norteamericano están frecuentemente muy bien escritas. Los autores saben cómo captar la atención de sus lectores: "Este es un agudo ataque a..." puede ser la primera frase de un artículo de derecho. El lector de inmediato es alertado. Además, los autores saben muy bien cómo plantear y sopesar su argumentación. Examinemos ahora un típico artículo de una revista de derecho europea. La exposición inicial del autor

${ }^{21}$ Mattei se refiere a ello como "la difusión por prestigio, basada en un proceso deliberado de admiración institucional que lleva a la recepción del derecho" (p. 6).

${ }^{22}$ Con la notable excepción de Lord Denning. 
será lo más neutral posible. Desde un punto de vista imparcial, el autor pasa a exponer y fundamentar una conclusión que se hace aparecer como completamente evidente. Tras el falso pretexto de parecer 'científica', la argumentación se realiza a través de métodos deductivos, lo que a su vez hace la lectura difícil y aburrida. Que los escritos académicos no tienen por qué ser aburridos para ser de calidad, es una lección que podemos aprender de los Estados Unidos.

El segundo ejemplo dice relación con el uso de denominaciones atractivas e innovadoras para nuevos conceptos jurídicos. En los glosarios norteamericanos, términos como blue sky law, ${ }^{23}$ lemon law, ${ }^{24}$ runaway shop, ${ }^{25}$ safe harbour clause, ${ }^{26}$ sunset clause, ${ }^{27}$ sweetheart contract $^{28}$ y yellow dog contract ${ }^{29}$ hacen el derecho fácil de retener. El inglés se convierte entonces en el latín del siglo XXI. Aun cuando esta tendencia no es completamente ajena a otras jurisdicciones, han sido los norteamericanos quienes la han desarrollado en mayor medida.

d) La preponderancia de los académicos norteamericanos está también relacionada con su gran número. El hecho de que existan tantos abogados en los Estados Unidos se debe, lógicamente, a que existen también muchas Facultades de Derecho. Aun cuando no todas ellas califican como 'top ten' a nivel mundial, existen suficientes de buena e incluso extraordinaria calidad. Si de cada cincuenta estudiantes uno se interesa en la academia, la potencialidad para formar académicos en el ámbito jurídico es enorme.

e) Finalmente, en relación con un argumento técnico, también planteado por Mattei, es el hecho que por ser los Estados Unidos un estado federal y descentralizado, los abogados norteamericanos deben ocupar una parte considerable de su tiempo en lidiar con temas relativos a la jurisdicción aplicable al caso concreto y a los conflictos de leyes. Esta circunstancia los capacita de mejor manera para participar más fácilmente que abogados de otras jurisdicciones en el discours internacional.

${ }^{23}$ Aquella ley destinada a regular la oferta y venta de instrumentos negociables, para proteger al público de fraudes.

${ }^{24}$ Aquella ley que otorga el derecho del consumidor a dejar sin efecto el contrato en razón de haber tenido que devolver repetidamente el bien objeto del contrato por fallas en la calidad del mismo. El nombre tiene su origen en la denominación coloquial de ‘lemon’ para aquellas personas con carácter agrio o poco amistoso.

${ }^{25}$ Término usado para referirse a empresas que constantemente cambian de ubicación geográfica para efectos de evadir leyes o regulaciones, especialmente en relación al ámbito laboral.

${ }^{26}$ Disposición legal o reglamentaria por la cual se excluye o limita la responsabilidad de una parte, que ha actuado de buena fe y ha adoptado las medidas preventivas fijadas en la misma norma para evitar el daño.

${ }^{27}$ Aquella disposición en virtud de la cual una ley pierde su vigencia transcurrido un cierto número de años.

${ }^{28}$ Aquel contrato que establece beneficios mutuos para el empleador y el sindicato en cuanto organización, pero en desmedro de los trabajadores que lo integran.

${ }^{29}$ Acuerdo entre empleador y trabajador, por el cual este último se compromete, como condición para mantener su empleo, a no afiliarse a un sindicato. 


\section{Desventajas e inconvenientes de la influencia del derecho NORTEAMERICANO}

La influencia del derecho y pensamiento jurídico estadounidense no está exenta de inconvenientes, de mayor o menor relevancia. Entre los más importantes podemos señalar: a) La renuencia de la teoría jurídica norteamericana a aceptar ideas provenientes de países distintos a los Estados Unidos o que no pertenezcan al sistema del common law; b) el clientelismo al que están sujetas algunas escuelas de pensamiento norteamericanas $y$, finalmente, c) la fuga de capital y cerebros que implica el hecho que muchos talentosos jóvenes abogados se decidan por realizar sus estudios de postgrado en los Estados Unidos. A continuación desarrollaré tales ideas.

a) La doctrina jurídica norteamericana no siempre está abierta a la consideración o influencia de argumentos foráneos. Los juristas norteamericanos ven algunas veces al mundo entero como parte del common law. Ello puede ser entendible desde un punto vista lingüístico, ya que la mayoría de las veces que los norteamericanos se enfrentan a ideas extranjeras se trata de obras provenientes del mundo de habla inglesa, el cual en términos jurídicos pertenece mayormente al common law. Sin embargo, lo anterior implica que argumentos o ideas provenientes de otras jurisdicciones son simplemente ignorados por la academia norteamericana.

b) Estados Unidos, así como también algunos otros países como Italia, sufre de clientelismo. Aquellos que no pertenecen a determinada escuela de pensamiento simplemente no son citados ni menos promovidos en el ámbito académico. Hasta cierto punto, es el caso del análisis económico del derecho, pero la escuela del estudio crítico del derecho es aún un mejor ejemplo: sus principales exponentes no niegan en público que buscan activamente que sus protegidos sean designados en cargos importantes. Quizás ello es inherente a la naturaleza humana, pero desde el punto de vista de los intereses de la sociedad en general es una consecuencia desafortunada.

c) Desde un punto de vista práctico, el hecho que los estudiantes de países en desarrollo elijan a las Facultades de Derecho de los Estados Unidos para proseguir sus estudios de postgrado implica en algunos casos una clara desventaja para sus países de origen. Tales Facultades reciben importantes beneficios económicos a cambio, y sacan provecho al mismo tiempo de algunos de los más brillantes talentos jurídicos. Es frecuente que un joven autor destaque orgullosamente en sus publicaciones su LLM obtenido en Harvard, pero ¿qué tan frecuente es que suceda lo mismo cuando el grado se ha obtenido en Giessen o Valencia?

\section{QUÉ HACER}

Ante el avance de la cultura jurídica norteamericana, ¿qué puede hacer el resto del mundo? ¿Debemos permanecer como simples observadores? Por supuesto que no. Ante todo, debemos aprender de los Estados Unidos. Segundo, debemos ser conscientes de nuestras fortalezas. Tercero, si debemos combatir a los norteamericanos, debemos 
hacerlo a través de organizaciones regionales. Cuarto, las ventajas lingüísticas de los angloparlantes deben ser neutralizadas. A continuación expondré estas ideas.

Primero, es evidente que debemos aprender de los norteamericanos. Debemos convertir nuestras tradicionales universidades en grandes instituciones de conocimiento. En Europa occidental sólo 10 a 20 facultades pueden plantearse en términos similares a las principales facultades norteamericanas. El cuerpo académico de tales universidades debería enfocarse en la investigación y el desarrollo de nuevas teorías antes que dedicar su tiempo a explicar lo que los tribunales y el legislador están haciendo. Además, deberían preocuparse de presentar su trabajo en un formato accesible. De gran relevancia es la elección de los símbolos adecuados para exponer las ideas.

Segundo, debemos reforzar y sacar partido a nuestras propias ventajas. No tener el inglés como idioma nativo puede ser una dificultad, pero también una ventaja. Ello nos habilita para leer y estudiar trabajos en latín, francés, alemán, italiano, español, entre otros idiomas. De este modo, podemos estudiar sistemas jurídicos de Derecho Civil (vigentes por cierto en más de la mitad del mundo) en su idioma original. Esta ventaja idiomática otorga a los no-estadounidenses una gran prerrogativa en la ciencia jurídica mundial.

Tercero, cuando debamos debatir o contrarrestar el derecho y las teorías jurídicas norteamericanas, no debemos hacerlo desde un punto de vista nacional, sino regional o continental. No ha sido el derecho o la doctrina de Texas o Nueva York los que han conquistado el mundo, sino el derecho y la doctrina estadounidense. De la misma manera, no es el derecho de Luxemburgo el que va a prevalecer sobre el de los Estados Unidos. Incluso, sistemas jurídicos como el francés o el alemán tendrán dificultades si enfrentan tal desafío de manera aislada. Sin embargo, tal posibilidad es real si actúan unidos bajo la Unión Europea. Por ello es tan importante que la Unión Europea esté desarrollando una política de armonización del derecho privado. Dicho ejemplo debería ser seguido por otras organizaciones regionales (como OHADA o MERCOSUR). Una anécdota personal permite ilustrar lo poderosa que como instrumento de difusión del derecho se ha convertido la Unión Europea.

Al momento del derrumbe del bloque comunista en la ex Unión Soviética y en Europa oriental, se encontraba como profesor visitante de la Facultad de Derecho de la Universidad de Utrecht un colega estadounidense. Al enterarse de la caída del Partido Comunista, nuestro colega norteamericano voló (literalmente) de inmediato a Moscú para promover entre los rusos sus ideas acerca de la forma de democracia requerida para la implementación de un sistema judicial basado en jurados. Más tarde, muchos otros norteamericanos se dirigieron a los países de Europa central y oriental para difundir y ofrecer las soluciones jurídicas estadounidenses a todo tipo de materias, desde derecho tributario hasta la implementación del artículo 9 del Uniform Commercial Code. Para su decepción, a la fecha muchos de los ex países comunistas habían ya suscrito acuerdos de cooperación en temas jurídicos con la Unión Europea. Ello ha tenido como consecuencia que tales países incorporen en sus sistemas jurídicos la normativa legal de la Unión Europea, el denominado acquis communautaire, y no el derecho de los Estados Unidos. 
Finalmente, en caso de ser necesario enfrentarse al derecho norteamericano, la ventaja idiomática puede ser neutralizada. Dos ejemplos demuestran que ello es posible. Primero, en los casos en que el inglés se utiliza como idioma exclusivo en el debate académico o la práctica comercial, puede recurrirse a terminología y expresiones que no tienen un significado técnico-jurídico preciso para los estadounidenses. Al contrario, si los términos técnicos que se utilizan son los propios del derecho norteamericano, se otorga a los estadounidenses una ventaja enorme, ya que sólo ellos conocen y manejan con precisión el significado e implicancias precisas de tal terminología. En cambio, el uso de una terminología 'neutral' deja a los estadounidenses y al resto del mundo en una posición más pareja. Ello es lo que la Comisión en Derecho Contractual Europeo (la Comisión Lando), al discutir y redactar los Principios de Derecho Europeo de Contratos, decidió hacer.

El segundo ejemplo también dice relación con el trabajo de la Comisión Lando. Aunque especialmente en la última década la mayoría de las sesiones de la comisión se realizaron exclusivamente en inglés, el francés se mantuvo como segundo idioma oficial. Siempre que debía redactarse una norma, la regla era que se hiciera también en francés. El resultado de ello es que, ante dudas de interpretación de los Principios, tanto el texto en inglés como el en francés se consideran auténticos y deben ser examinados. Así, la ventaja del angloparlante se equilibra con la del hablante francés. En este sentido, un número variado de idiomas oficiales, como en el caso de las Naciones Unidas, servirá para hacer aún más democrático el acceso a los textos.

\section{Conclusiones}

En este artículo he desarrollado algunas reflexiones acerca del impacto del derecho y el pensamiento jurídico norteamericano en el mundo contemporáneo. Los estadounidenses deberían ser elogiados por la calidad de sus teorías jurídicas y por la forma en que las exponen. Los métodos para difundir tales teorías, sin embargo, no siempre son sujetos a un escrutinio crítico. En más de una ocasión, los académicos norteamericanos dan por sentado que sus teorías serán igualmente válidas al aplicarse a otros sistemas jurídicos. Donde debemos superarlos es en sus ventajas idiomáticas. La tendencia estadounidense a convertir el inglés en el idioma global debe contrarrestarse, y si ello no es posible, su predominio sobre el idioma inglés debe ser impugnado.

\section{BIBLIOGRAFÍA}

Glenn, H. P., Legal Traditions of the World, Oxford U.P., Oxford, 2004.

Hesselink, M. W., The New European Private Law/Essays on the Future of Private Law, The Hague, Kluwer, 2002.

Hondius, E., "The Supremacy of Western Law", en C. de Ligt et al. (Ed.), Viva vox iuris Romanil Essays in honour of Johannes Emil Spruit, Gieben, Amsterdam 2002. 
Kennedy, D., A Critique of Adjudication (fin de siècle), Cambridge, Mass., 1997.

MAtTei, U., "A Theory of Imperial Law: A Study on U.S. Hegemony and the Latin Resistance", Global Jurist Frontiers, vol. 3, issue 2, (2003), disponible en http://www.bepress.com/gj/ frontiers/vol3/iss2/art1/\#.

Reimann M., "Product Liability in a Global Context: The Hollow Victory of the European Model", en European Review of Private Law, 2003. 